\title{
Protection against methylglyoxal-derived AGEs by regulation of glyoxalase 1 prevents retinal neuroglial and vasodegenerative pathology
}

\author{
A. K. Berner • O. Brouwers • R. Pringle • I. Klaassen • L. Colhoun • C. McVicar • \\ S. Brockbank • J. W. Curry • T. Miyata • M. Brownlee • R. O. Schlingemann • \\ C. Schalkwijk • A. W. Stitt
}

Received: 19 September 2011 /Accepted: 7 November 2011 /Published online: 6 December 2011

(C) Springer-Verlag 2011

\begin{abstract}
Aims/hypothesis Methylglyoxal (MG) is an important precursor for AGEs. Normally, $\mathrm{MG}$ is detoxified by the glyoxalase (GLO) enzyme system (including component enzymes GLO1 and GLO2). Enhanced glycolytic metabolism in many cells during diabetes may overpower detoxification capacity and lead to AGE-related pathology. Using a transgenic rat model that overexpresses GLO1, we investigated if this enzyme can inhibit retinal AGE formation and prevent key lesions of diabetic retinopathy. Methods Transgenic rats were developed by overexpression of full length GLO1. Diabetes was induced in wild-type (WT) and GLO1 rats and the animals were killed after 12 or 24 weeks of hyperglycaemia. $N^{\varepsilon}$-(Carboxyethyl)lysine (CEL), $N^{\varepsilon}$-(carboxymethyl)lysine (CML) and MG-derived-
\end{abstract}

A.K. Berner, O. Brouwers and R. Pringle contributed equally to this study.

Electronic supplementary material The online version of this article (doi:10.1007/s00125-011-2393-0) contains peer-reviewed but unedited supplementary material, which is available to authorised users.

A. K. Berner $\cdot$ R. Pringle $\cdot$ L. Colhoun $\cdot$ C. McVicar $\cdot$

S. Brockbank $\cdot$ J. W. Curry $\cdot$ A. W. Stitt $(\bowtie)$

Centre for Vision and Vascular Science, Queen's University

Belfast, Royal Victoria Hospital,

Belfast BT12 6BA, Northern Ireland, UK

e-mail: a.stitt@qub.ac.uk

O. Brouwers $\cdot$ C. Schalkwijk

Department of Internal Medicine, Universiteit Maastricht,

Maastricht, the Netherlands

T. Miyata

United Centers for Advanced Research

and Translational Medicine (ART),

Tohoku University Graduate School of Medicine,

Miyagi, Japan hydroimidazalone-1 (MG-H1) were determined by immunohistochemistry and by ultra-performance liquid chromatography tandem mass spectrometry (UPLC-MSMS). Müller glia dysfunction was determined by glial fibrillary acidic protein (GFAP) immunoreactivity and by spatial localisation of the potassium channel Kir4.1. Acellular capillaries were quantified in retinal flat mounts.

Results GLO1 overexpression prevented CEL and MG-H1 accumulation in the diabetic retina when compared with WT diabetic counterparts $(p<0.01)$. Diabetes-related increases in Müller glial GFAP levels and loss of Kir4.1 at the vascular end-feet were significantly prevented by GLO1 overexpression $(p<0.05)$ at both 12 - and 24 -week time points. GLO1 diabetic animals showed fewer acellular capillaries than WT diabetic animals $(p<0.001)$ at 24 weeks' diabetes.

I. Klaassen $\cdot$ R. O. Schlingemann

Department of Ophthalmology, Academic Medical Center,

University of Amsterdam,

Amsterdam, the Netherlands

R. O. Schlingemann

Department of Clinical and Molecular Ophthalmogenetics, The Netherlands Institute for Neuroscience, Royal Netherlands Academy of Arts and Sciences (KNAW),

Amsterdam, the Netherlands

M. Brownlee

Einstein Diabetes Research Center,

Albert Einstein College of Medicine,

New York, NY, USA 
Conclusions/interpretation Detoxification of MG reduces AGE adduct accumulation, which, in turn, can prevent formation of key retinal neuroglial and vascular lesions as diabetes progresses. MG-derived AGEs play an important role in diabetic retinopathy.

Keywords AGEs · Diabetic complications · Diabetic retinopathy - Glyoxalase $1 \cdot$ Methylglyoxal · Pathogenesis . Retina

$\begin{array}{ll}\text { Abbreviations } & \\ \text { 3-DG } & \text { 3-Deoxyglucosone } \\ \text { AR } & \text { Aldose reductase } \\ \text { CEL } & N^{\varepsilon} \text {-(Carboxyethyl)lysine } \\ \text { CML } & N^{\varepsilon} \text {-(Carboxymethyl)lysine } \\ \text { GFAP } & \text { Glial fibrillary acidic protein } \\ \text { GLO } & \text { Glyoxalase } \\ \text { GO } & \text { Glyoxal } \\ \text { GSH } & \text { Glutathione } \\ \text { MG } & \text { Methylglyoxal } \\ \text { MG-H1 } & \text { MG-derived-hydroimidazalone-1 } \\ \text { RAGE } & \text { Receptor for AGEs } \\ \text { UPLC-MSMS } & \text { Ultra-performance liquid chromatography } \\ & \text { tandem mass spectrometry } \\ \text { WT } & \text { Wild-type }\end{array}$

\section{Introduction}

Diabetic retinopathy is a complex condition with a multifactorial pathogenesis. Among several hyperglycaemia-mediated pathogenic mechanisms that may contribute to this diabetic complication is the formation and accumulation of AGEs [1]. Clinical studies have demonstrated that serum levels of $N^{\varepsilon}$ (carboxy-methyl)lysine (CML), pentosidine and methylglyoxal (MG)-derived hydroimidazolone-1 (MG-H1) are associated with disease progression [2-4]. However, serum AGEs can be variable and evidence suggests that intracellular and matrix-immobilised AGEs are more robust biomarkers [1]. For example, Genuth et al. have demonstrated that crosslinking AGEs on long-lived skin proteins are significantly associated with progression of diabetic retinopathy [5]. Beyond clinical correlates, AGEs and related advanced lipoxidation end-products (ALEs) accumulate in the retinal vasculature and Müller glia during diabetes [6-8], and AGE inhibitors show efficacy in reducing retinal lesions in diabetic animal models [8-11].

AGEs can form directly from the reaction of glucose with amino groups or from the reaction with $\alpha$-oxoaldehydes such as glyoxal (GO), MG and 3-deoxyglucosone (3-DG). These highly reactive intermediates arise from both chemical and metabolic pathways and can lead to rapid adduct formation on lysine and arginine residues [12]. $\alpha$-Oxoaldehydes occur at elevated levels in cells exposed to high glucose and also in diabetic serum, and are probably the most important source of intra- and extracellular AGEs [13, 14]. For example, MG can give rise to $N^{\varepsilon}$-(carboxyethyl)lysine (CEL), argpyrimidine and MG-H1, which have been identified at elevated levels in diabetic tissues $[14,15]$.

$\alpha$-Oxoaldehydes are a normal product of metabolism, and cells possess a range of endogenous enzyme systems that 'detoxify' these AGE precursors. For example, a glutathionedependent glyoxalase complex (formed from glyoxalase I [GLO1] and glyoxalase II [GLO2] components) protects cells by converting GO and MG to D-lactate [16]. The capacity of this enzyme system to limit AGEs is demonstrated in endothelial cells which were transfected to overexpress GLO-1 and subsequently showed less accumulation of MG-derived adducts [17]. This was accompanied by protection against high glucose-mediated dysfunction [17, 18]. Overexpression of GLO1 in diabetic rats has been shown to be protective against AGE formation and oxidative stress in muscle [19], and also prevent abnormalities in endothelium-dependent vasorelaxation [20]. Likewise, regulation of $G L O 1$ activity in cells can prevent diabetes-related cell dysfunction [21, 22].

MG-derived AGEs are important in diabetic retinopathy. It has been demonstrated that retinal levels of MG-H1 increase by $279 \%$ following 24 weeks of diabetes [23], and that MG-H1 correlates with diabetic retinopathy [3]. In retinal cells, MG induces retinal pericyte dysfunction [24] and $G L O 1$ has been shown to protect against premature death of pericytes and endothelial cells following high glucose exposure [22, 25].

Given that MG-derived adducts are important clinically and that prevention of this pathway has therapeutic potential, we hypothesise that MG-derived AGEs play a key role in neuroglial and microvascular degenerative pathology during the early and intermediate stages of diabetic retinopathy. If this is the case, modulation of the glyoxalase detoxification system could be protective and prevent formation of key retinal lesions during diabetes.

\section{Methods}

Diabetic rat model Rats transgenic for human GLO1 were developed by overexpression of the full-length GLO1 cDNA under control of the cytomegalovirus enhancer/chicken $\beta$-actin promoter system and transgene expression in various tissues was confirmed by PCR. GLO1-overexpressing rats were crossed with wild-type (WT) Wistar rats to obtain enough GLO1 progeny for the experiment. The rats were obtained from the Nippon Seibutsu Zairyo Center (Saitama, Japan) and studies using these animals have been recently published [19, 20]. All 
animal studies were carried out in accordance with the Guide for the Care and Use of Laboratory Animals of the National Institutes of Health. All experiments involving rats were reviewed and approved by the Ethics Committee for Animal Care and Use of Maastricht University, the Netherlands.

A total of 55 animals were included in the study (31 WT and 24 GLO1 rats). At the age of 10 weeks, both rat groups were rendered diabetic by a tail-vein injection of streptozotocin (STZ) $(65 \mathrm{mg} / \mathrm{kg}$ in citrate buffer for 3 months' duration of diabetes, and $45 \mathrm{mg} / \mathrm{kg}$ for 6 months' duration) to prevent severe illness. At 1 and 12 weeks post diabetes induction, blood glucose was measured and only rats with a blood glucose $>15 \mathrm{mmol} / \mathrm{l}$ at both time points were included in the study. Diabetes was maintained for 3 and 6 months prior to death.

Rat retinal immunohistology Enucleated eyes were fixed in $4 \%$ paraformaldehyde (PFA) for $30 \mathrm{~min}$, stored in PBS containing $0.1 \%$ sodium azide and shipped from the University of Maastricht, the Netherlands to Belfast for investigation. The posterior eye-cup was separated from the globes and the retinas then dissected into two. The half retinas from each sample population were immediately mounted in cryo-embedding compound (BDH, Poole, UK) in a bath of iso-pentane surrounded with dry ice. Samples were stored at $-20^{\circ} \mathrm{C}$ until used. Prior to sectioning, samples were allowed to equilibrate for approximately $20 \mathrm{~min}$ in a cryostat. Retinas were vertically sectioned at 12 or $20 \mu \mathrm{m}$ and then collected on Superfrost/Plus microscope slides (Menzel-Glaser, Braunschweig, Germany) and stored at $-20^{\circ} \mathrm{C}$ until use. The other half retinas were used as flat mount preparations (see Staining for acellular capillaries).

Frozen retinal sections were thawed at room temperature and then circled with a DAKO pen (DAKO, Glostrup, Denmark) to provide a barrier for the solutions applied to the sections. They were rehydrated by the application of $200 \mu \mathrm{l}$ of PBS $(3 \times 10 \mathrm{~min})$. Samples were blocked (PBS, $\mathrm{pH} 7.4,10 \%$ goat serum, $0.1 \%$ Tritron $\mathrm{X}-100$ ) for $1 \mathrm{~h}$ in a humidity chamber, followed by incubation in a range of primary antibodies including: MG-H1, GLO1 (BioMac, Leipzig, Germany), glial fibrillary acidic protein (GFAP) (Dako), CEL and CML (Cosmobio, Carlsbad, CA, USA), and Kir4.1 (Alomone, Jerusalem, Israel). All primary antibodies were diluted in blocking solution overnight at $4^{\circ} \mathrm{C}$. A series of $4 \times 10 \mathrm{~min}$ washes in PBS was followed by a 1-h incubation at room temperature in an appropriate alexa fluor secondary antibody in the dark (Molecular Probes, Paisley, UK). Sections were washed again $4 \times$ 10 min in PBS and later incubated with DAPI nuclear stain for $5 \mathrm{~min}$, followed by a series of $3 \times 5 \mathrm{~min}$ washes. Sections were coverslipped with vectashield (Vector Laboratories, Peterborough, UK). Control experiments were performed by addition of secondary antibody to observe the extent of non-specific antibody binding and secondary antibody autofluorescence.

Fluorescence was either visualised using a Nikon TE-2000 inverted microscope fitted with Nikon C1 confocal system (Nikon, Surrey, UK) or an epi-fluorescence microscope (Nikon Eclipse E400; Nikon). Images were taken from central regions of the retina. For each primary antibody used in the study, the sample with brightest fluorescence was measured first, and confocal settings were held constant when recording images of all subsequent samples. Images were processed and analysed using either ImageJ software (NIH, Bethesda, MD, USA), NIS Elements software (Nikon) or Velocity software (Mountain View, CA, USA). Immunofluorescence was measured by the intensity of the pixels above a threshold, which was defined as 4 SDs above the mean background fluorescence intensity measured from regions devoid of retinal tissue. A user-defined frame was drawn around each layer of the retina and the average fluorescence intensity above the threshold calculated. Either the left or the right eye was analysed for each rat. Six tissue sections were examined per retina.

Ultra-performance liquid chromatography tandem mass spectrometry analysis The AGE adducts CML, CEL and MG-H1 were measured in eyes and retina with ultraperformance liquid chromatography tandem mass spectrometry (UPLC-MSMS) as described in detail previously [19]. Liquid chromatography was performed at $30^{\circ} \mathrm{C}$ using an Acquity UPLC BEH C18, $1.7 \mu \mathrm{m}, 2.1 \times 100 \mathrm{~mm}$ column (Waters, Milford, MA, USA), and the Micromass Quattro Premier XE Tandem Mass Spectrometer (Waters) was used in electrospray-positive multiple reaction monitoring mode.

Quantitative PCR RNA isolation, reverse transcription and real-time quantitative PCR was performed on retinas isolated from the 3-month experimental groups. The approach was as described previously [26]. Transcripts investigated included $\operatorname{Veg} f, \operatorname{Tg} f \beta-1$ and -2 (also known as Tgfb1 and -2), Ctgf, Icam1, Gal3 and Timp1.

Acellular capillary quantification Retina flat mounts were prepared for immunofluorescence evaluation as previously described [27]. The retinal flat mounts were stained with biotinylated isolectin GS-IB4 overnight (Sigma, Dorset, UK) or for collagen IV immunoreactivity (Acris Antibodies, Germany). Appropriate ligand (streptavidin Alexa Fluor 488) and secondary antibody were used (Alexa Fluor 568 goat anti-rabbit IgG) (both from Molecular Probes). DAPI (Sigma) was also added to visualise the nuclear layers of the retina. Stained retinas were imaged using confocal microscopy (Eclipse TE2000-U; Nikon). Five regions were taken at $\times 20$ in the central and peripheral retina for collagen IV and lectin. 
Statistical analysis Data were expressed as the mean values \pm SEM. Statistical differences in the mean were assessed using the unpaired Student's $t$ test or ANOVA. All the statistical analyses were performed using Graph pad InStat 3.0 (GraphPad Software, San Diego, CA, USA).

\section{Results}

GLO1 expression in rat retina The diabetic status of these animals has been previously published [19]. The diabetic groups after both time periods were characterised by hyperglycaemia, polydipsia, hyperphagia, polyuria and decreased weight gain, irrespective of their transgenic status The GLO1 transgene had no influence on hyperglycaemic status or body weight [19]. GLO1 activity in various tissues has also been published indicating that there is a significant elevation of functional enzymatic activity when compared with WT controls [19]. The eyes of these animals in particular, demonstrated a $\sim 25$-fold increase in GLO1 activity [19]. GLO1 immunoreactivity in the retina was markedly elevated in the GLO1 transgenic rats and was ubiquitously expressed throughout the neural retina (Fig. 1a). Diabetes did not alter the nature of the transgene expression (Fig. 1a-c). The intense immunoreactivity in the
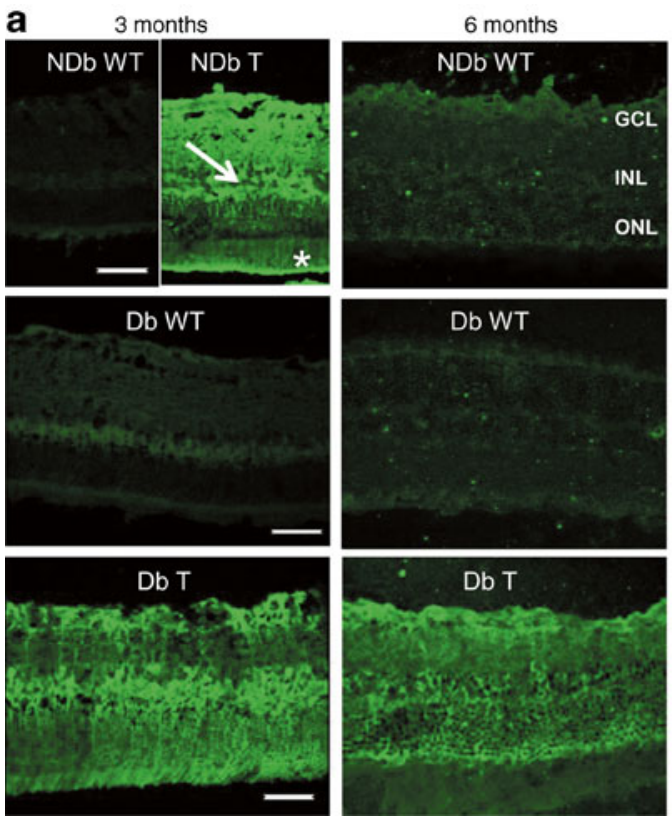

Fig. 1 GLO1 protein in 3-month and 6-month WT and GLO1transgenic rat retina. a Immunofluorescence labelling from nondiabetic wild-type (NDb WT), non-diabetic transgenic (NDb T), diabetic wild-type (Db WT) and diabetic GLO1 transgenic (Db T) rat retina $(n \geq 3)$. Transgenic animals exhibited higher protein levels of GLO1 throughout all layers of the retina (green), although there is particular intensity at the level of the inner nuclear layer (INL), inner limiting membrane (arrow) and external limiting membrane $(*)$. This demonstrates that the TG animals overexpress GLOI in the retina in a cell bodies of the inner nuclear layer in combination with vertically striated cell localisation from inner to outer retina indicated a high level of Müller glia expression (Fig. 1a). Overall there was no quantitative difference in GLOI levels during diabetes, although expression appeared less when GLO1 rats that had been diabetic for 6 months were compared with those diabetic for 3 months (Fig. $1 b$ and c). This suggests that expression of the transgene was reduced over time or that longer-term diabetes reduced GLO1 expression.

GLO1 overexpression reduces retinal AGEs during diabetes Various AGE adducts were assessed using a combination of immunohistochemistry and UPLC-MSMS approaches. AGE localisation has been previously demonstrated in the retina and it is established that many of these adducts show a considerable degree of accumulation in Müller glia during diabetes [28-30]. MG-derived CEL has not been previously reported in the retina and this adduct demonstrated increased immunoreactivity in the diabetic retina, even after 3 months of hyperglycaemia, and localisation was in a pattern consistent with Müller glia (Fig. 2a). In diabetic GLOI animals, CEL was markedly reduced and localised largely to an astrocyte-like pattern close to the internal limiting membrane (Fig. 2a). Immunolocalisation patterns for each

b
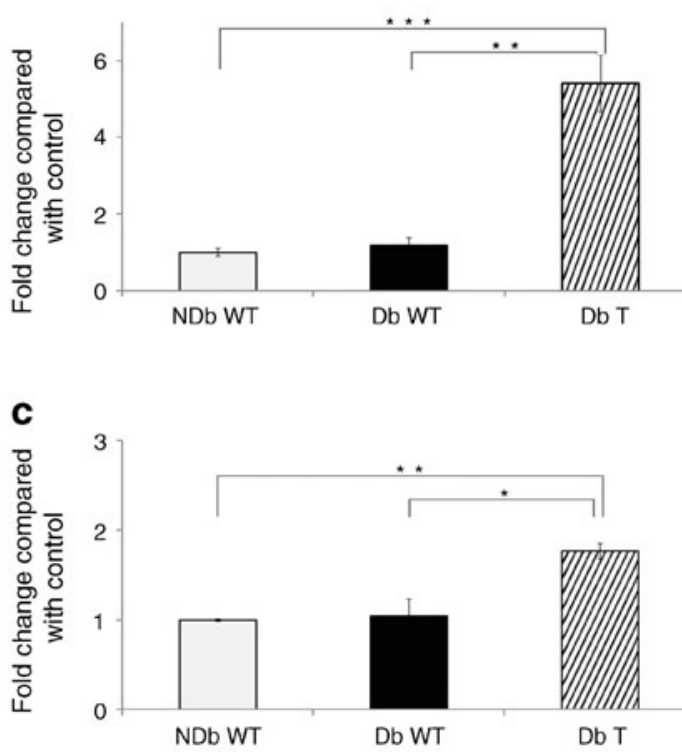

pattern suggesting particularly high levels in the Müller glia. b and c Quantification of retinal GLO1 protein levels for (b) 3- and (c) 6month diabetic rat groups and normalisation to control (NDb WT) levels demonstrates statistical differences between WT and GLOI transgenic rat retina. There is no difference in GLO1 levels between non-diabetic and diabetic WT animals $(\mathrm{a}-\mathrm{c})$. Scale bars $50 \mu \mathrm{m}$, magnification $\times 40,12 \mu \mathrm{m}$ cryostat sections. GCL, ganglion cell layer; ONL, outer nuclear layer. ${ }^{*} p<0.1,{ }^{* *} p<0.01,{ }^{* * *} p<0.001$ 
a

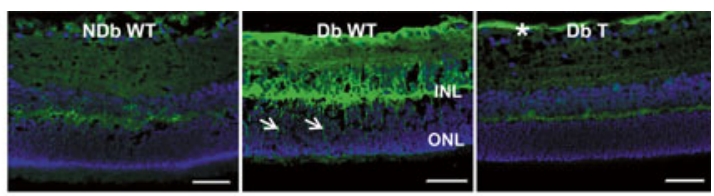

b
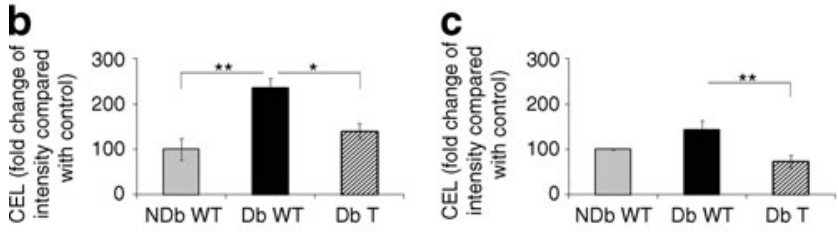

d

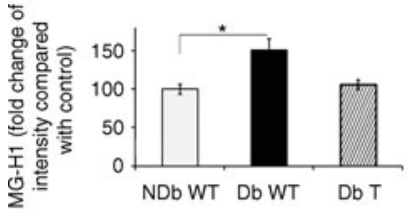

f
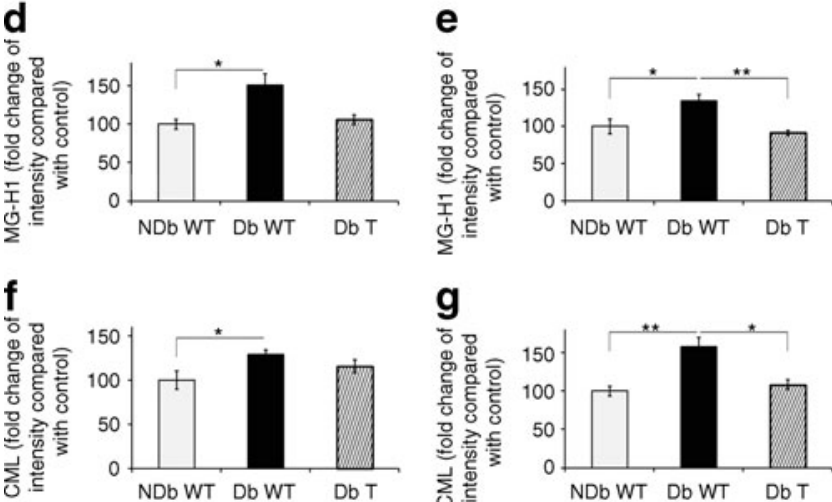

g

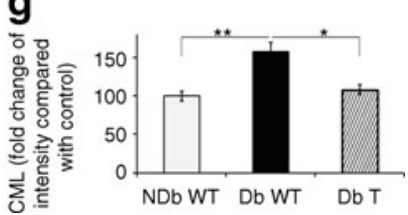

Fig. 2 AGE immunoreactivity is increased in diabetic retina and are reduced by $G L O 1$ overexpression. a CEL immunoreactivity was markedly increased in the diabetic retina when compared with nondiabetic controls. The localisation pattern of this AGE was consistent with Müller glia showing fluorescence intensity at the inner nuclear layer (INL), inner limiting membrane (arrow) and cytoplasmic processes (arrows) through the outer nuclear layer (ONL). CEL was markedly reduced in diabetic GLO1 animals and localised largely to an astrocyte-like pattern close to the internal limiting membrane $\left(^{*}\right)$. Scale bars $50 \mu \mathrm{m}$, magnification $\times 40$. Nuclei counterstained with DAPI. CEL (b,c), MG-H1 (d,e) and CML (f,g) immunoreactivity was quantified in retinal sections at 3 months $(\mathbf{b}, \mathbf{d}, \mathbf{f})$ and 6 months $(\mathbf{c}, \mathbf{e}, \mathbf{g})$. The total intensity of the adducts occurring in the diabetic WT (Db WT) and diabetic GLO1 transgenic (Db T) rat retinas was analysed and normalised against controls (NDb WT). Diabetic retinas showed higher AGE levels, whereas GLO1 overexpression reduced the AGE burden in diabetic rats. ${ }^{*} p<0.1,{ }^{*} p<0.01$

of the three AGE adducts (CEL, CML and MG-H1) were similar, and, on quantification, CML and MG-H1 were also significantly increased in the diabetic retina at both 3 and 6 months' post induction $(p<0.05-0.01)$, and presence of the GLO1 transgene reduced these levels to that of non-diabetic controls (Fig. 2b-g).

AGEs quantified using UPLC-MSMS analysis of whole eye homogenates following 3 or 6 months of diabetes showed a pattern that was comparable with that obtained by immunohistochemistry. There was a significant increase in CEL, MG-H1 and CML in the diabetic retina compared with non-diabetic controls for both 3- and 6-month diabetic animals $(p<0.05-0.001)$. Overexpressing GLO1 diabetic retina appeared to reduce the accumulation of these AGEs in the 6-month group, although not to levels of the non-diabetic controls (Fig. 3a-f). For animals that were 3 months' diabetic only, it was possible to analyse isolated retina by UPLCMSMS (electronic supplementary material [ESM] Fig. 1). Although the data did not show significance between the groups, there were apparent trends that corresponded to the retinal immunohistochemistry and whole eye UPLC-MSMS results (compare Figs 2, 3 and ESM Fig. 1).

GLO1 overexpression prevents Müller glia dysfunction during diabetes In the non-diabetic retina from both WT and GLO1 rats, GFAP was localised to the astrocytes (Fig. 4a). Diabetes induced a strong upregulation of GFAP in both astrocytes and retinal Müller glia $(p<0.05)$, and there was a small but significant diminution of this protein after 3 months' diabetes in the GLO1 rats, an effect that became more pronounced after 6 months' diabetes $(p<0.05-0.001)$ (Fig. 4b and c). Another robust diabetesmediated response by the retinal Müller glia is the mislocalisation of the weakly inward rectifying $\mathrm{K}^{+}$channel Kir 4.1, which is typically localised in Müller glia end-feet and surrounding blood vessels [31]. In diabetic retina, Kir4.1 becomes less localised at the interface with the retinal blood vessels and reduced at the ILM [6, 31]. Following both 3 and 6 months' diabetes, this Kir4.1 response was also observed in the current study (Fig. 5). Overexpression of GLO1 during diabetes maintained the vascular and Müller glia end-feet localisation (Fig. 5). a

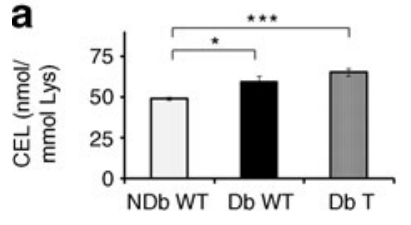

C

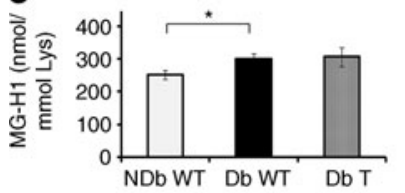

e

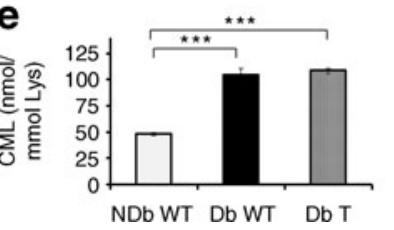

b

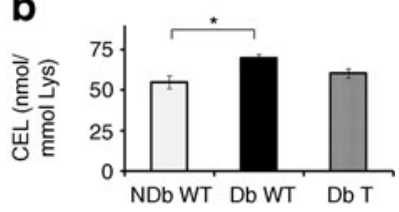

d

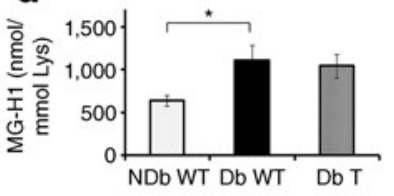

f

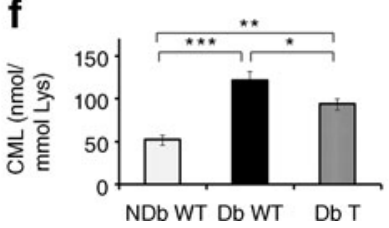

Fig. 3 AGE quantification in diabetic ocular tissue by UPLC-MSMS. AGEs in whole eye homogenates were analysed using UPLC-MSMS. The levels of CEL (a,b), MG-H1 (c,d) and CML (e,f) show similar trends with enhanced concentrations after 3 months' (a,c,e) and 6 months' (b,d,f) diabetes compared with non-diabetic controls. GLOI overexpressing diabetic animals show reduced AGE levels but only after 6 months. ${ }^{*} p<0.05 ; * * p<0.01 ; * * * p<0.001$. Db, diabetic; NDb, non-diabetic; $\mathrm{T}$, transgenic 

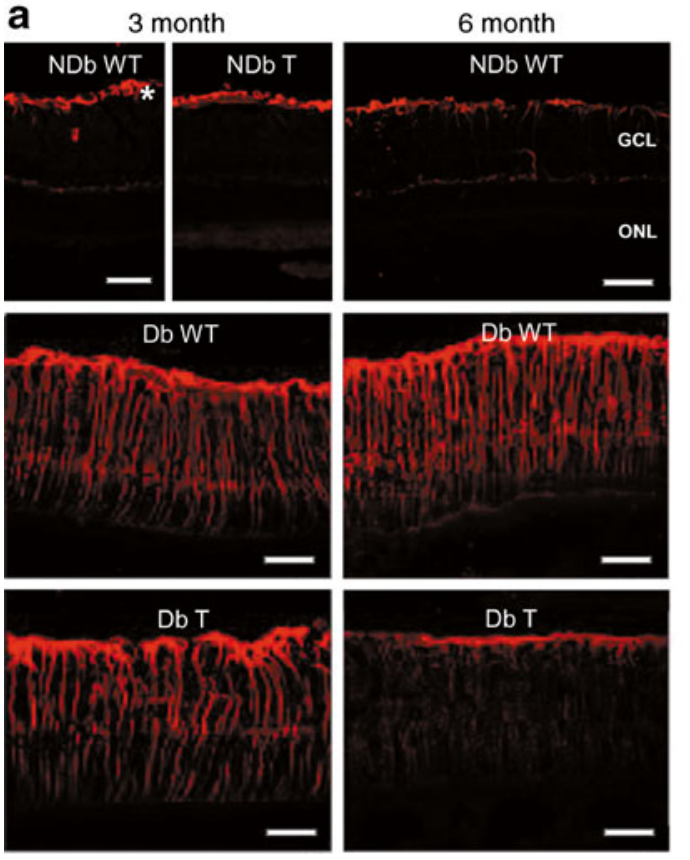

Fig. 4 GLO1 regulates GFAP levels in retinal Müller glia during diabetes. a GFAP in non-diabetic WT and transgenic rat retina is localised mainly in astrocytes (*). Following 3 and 6 months of diabetes, GFAP is strongly produced in the retinal Müller glia. Overexpression of GLO1 reduces this diabetes-mediated response, especially after more prolonged periods of hyperglycaemia. Scale bars $50 \mu \mathrm{m}$, magnification $\times 40,12 \mu \mathrm{m}$ cryostat sections. GCL, ganglion cell layer; ONL, outer nuclear layer. $\mathbf{b}$ and $\mathbf{c}$ Retinal GFAP levels were

GLO1 overexpression in diabetic animals alters retinal expression of key transcripts Quantitative PCR data demonstrated that a range of transcripts was altered during diabetes (Fig. 6). In the WT animals, diabetes induced a significant upregulation of Gal3, Ctgf, Timpl and Icam 1 compared with non-diabetic controls. By contrast, transgenic animals with diabetes did not show this response and it appeared that high GLO1 attenuated these diabetesinduced responses (Fig. 6). Vegfa was not altered by either diabetes or the presence of the GLO1 transgene. Tgf $\beta-1$ and -2 mRNA expression was shown to be downregulated in the GLO1 diabetic retina compared with diabetic WT controls (Fig. 6).

GLO1 protects against capillary closure Diabetic rats typically show formation of acellular capillaries at around 6 months' post induction [29]. The current study identified collagen IV-positive vascular basement membrane tubes representative of the entire vascular tree in combination with isolectin, which labelled endothelial cells. Strands positive for collagen IV and negative for isolectin indicated acellular capillaries (Fig. 7). Diabetes induced a significant increase in capillary dropout in both the peripheral and central retina, with the latter showing greater levels of pathology (Fig. 7). GLO1 overexpression in diabetic rats b

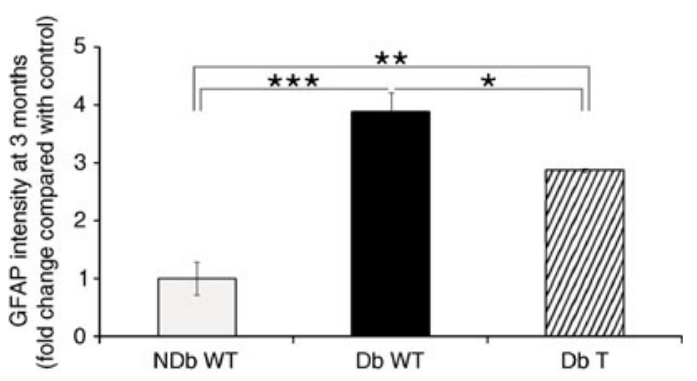

C

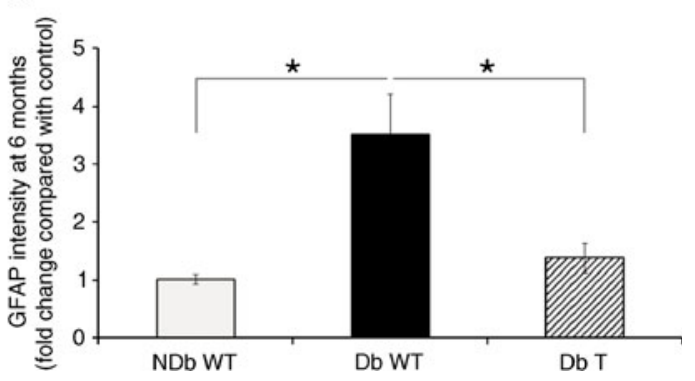

highest in the diabetic WT retina (Db WT) when compared with nondiabetic WT animals (NDb WT). GFAP was significantly reduced in the diabetic GLO1 transgenic retina (Db T) in both 3-month (b) and 6-month (c) diabetic groups. No significant differences could be observed between NDb WT and $\mathrm{Db} \mathrm{T}$ groups in terms of GFAP levels after 6 months. Data were normalised against NDb WT values. ${ }^{*} p<0.1,{ }^{* *} p<0.01, * * * p<0.001$

significantly prevented acellular capillary formation and there were no differences between these treated diabetics in comparison with non-diabetic control groups (Fig. 7).

\section{Discussion}

The relationship between raised intracellular $\mathrm{MG}$, rapid AGE formation and the detoxifying potential of the glyoxalase system has been the focus of intensive study in the area of diabetic complications and ageing [17, 18, 32]. In the context of diabetic retinopathy, the current study has demonstrated that GLO1 overexpression in diabetic rats prevents hyperglycaemia-induced formation of MG-derived AGEs in the neural retina and prevents Müller glia dysfunction and protects against capillary degenerative pathology. This follows on from previous reports in diabetic rats in which GLO1 overexpression protected against elevated tissue and systemic circulating levels of AGEs and oxidative stress markers [19], and also prevented hyperglycaemia-mediated impairment of arterial vasorelaxation [20].

Accumulation of AGEs in the diabetic retina is an established phenomenon and their inhibition can effectively prevent key aspects of diabetic retinopathy [8, 9] [33]. 


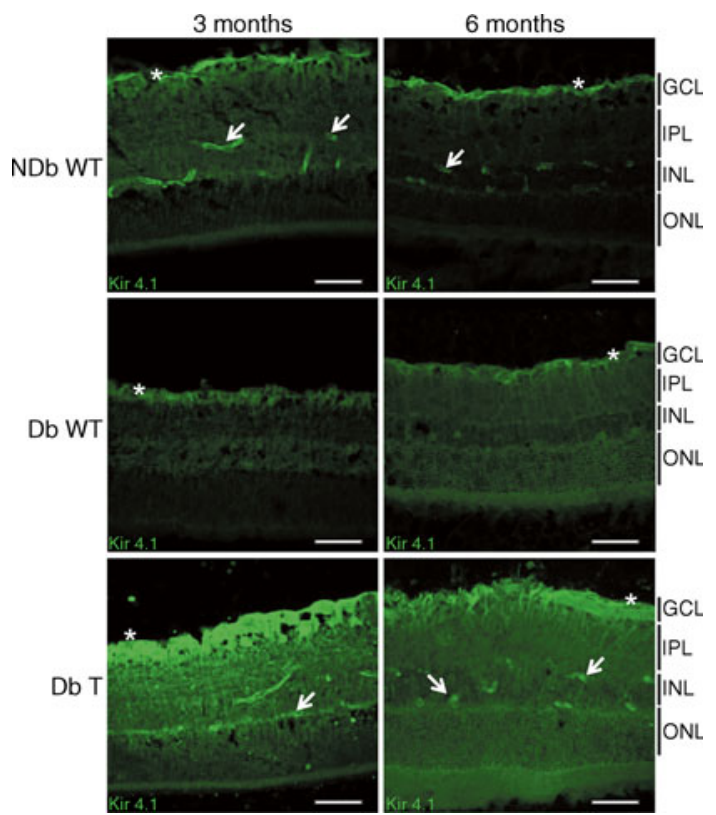

Fig. 5 Diabetes-mediated mis-localisation of Kir4.1 is prevented by GLO1 overexpression. In normal retina, the inwardly rectifying channel Kir4.1 (green) is localised at the end-feet of Müller glia at the internal limiting membrane (ILM) $\left(^{*}\right)$ and the perivascular interface (arrows). In diabetic retina, Kir4.1 is no longer visible around the retinal vasculature but is expressed more widely throughout the Müller glia cytoplasm (compare Db WT with the other groups). GLO1 overexpression in the diabetic retina reduced this diabetes-mediated cellular localisation defect (compare DB T with Db WT). Blue: nuclear stain DAPI, magnification $\times 20$, scale bar $100 \mu \mathrm{m}$, $20 \mu \mathrm{m}$ cryostat sections ( 3 month) and $14 \mu \mathrm{m}$ sections (6 month). GCL, ganglion cell layer; INL, inner nuclear layer; IPL, inner plexiform layer; ONL, outer nuclear layer
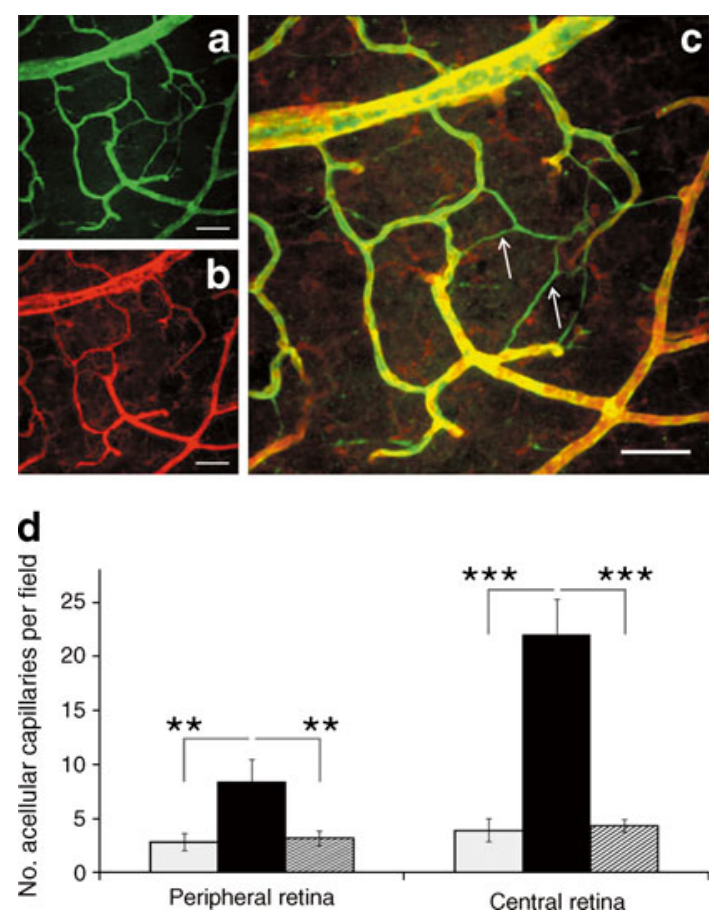

Fig. $7 \mathrm{GLOl}$ overexpression prevents capillary degeneration during diabetic retinopathy. Six months' diabetes causes acellular capillary formation in the retina and this is demonstrated by confocal microscopy of flat-mounted retina. The images show an example of a Db WT retina stained for collagen IV (basement membrane; green) (a) and isolectin (viable endothelium; red) (b). The combined image of (a) and (b) clearly demonstrates the presence of empty basement membranes without endothelial cells, quantified as 'acellular capillaries' (arrows) (c). d GLO1 overexpression in diabetic retinas significantly reduces the amount of acellular capillaries in the peripheral and central retina. Grey bars, NDb WT; black bars, Db WT; hatched bars, Db T. Scale bars $50 \mu \mathrm{m}$, magnification $\times 20$. $* * p<0.01, * * * p<0.001$

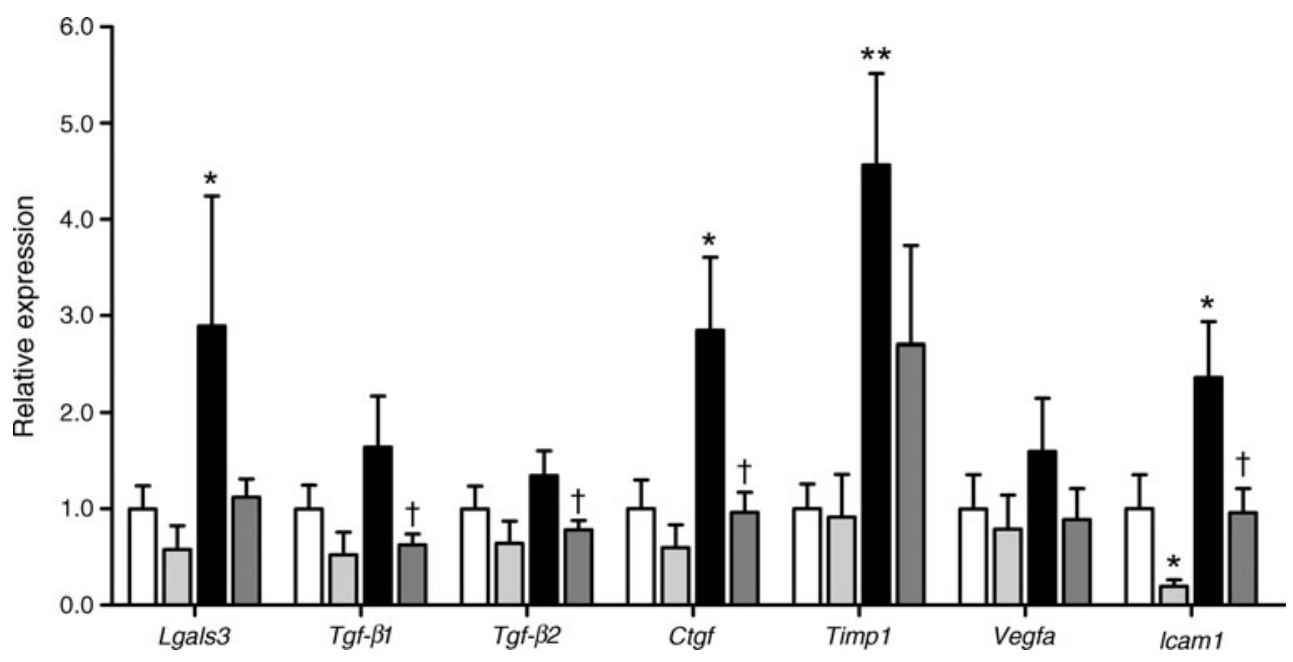

Fig. 6 Retinal mRNA expression levels of genes regulated during diabetes. The graph shows fold change between the non-diabetic wildtype rats (NDb WT), non-diabetic transgenic GLO1 rats (NDb T), wild-type rats with 3 months' diabetes (Db WT) and transgenic GLO1 rats with 3 months' diabetes ( $\mathrm{Db} \mathrm{T}$ ). White bars, NDb WT; light grey bars, NDb T; black bars, Db WT; dark grey bars, Db T. * $p<0.05$ for difference between experimental group and NDb WT rats; $* * p<0.01$ for difference between experimental group and NDb WT rats; ${ }^{\dagger} p<0.05$ for difference between diabetic transgenic and Db WT animals 
Although AGEs form in the retina as diabetes progresses, their precise derivation and the relative importance of various adducts remains unclear. The efficacy of benfotiamine in preventing lesions of diabetic retinopathy by addressing hyperglycaemia-mediated oxidative stress and associated elevations of intracellular MG suggests that this $\alpha$-oxoaldehyde is an important source of AGEs [11]. To further dissect the importance of MG-derived AGEs in diabetic retinas, the current study has combined immunohistochemistry and UPLC-MSMS to show that CEL, CML and MG-H1 are elevated when compared with non-diabetic controls, and that these adducts appear to accumulate in the retina, particularly in Müller glia. Furthermore, the evidence that $G L O 1$ overexpression can prevent retinal cell dysfunction and death indicates that MG and GO are not only major precursors for AGEs in the diabetic retina but also that they impact on disease progression.

MG-derived adduct formation also occurs alongside the 'classic' Maillard chemistry and other pathways such as myeloperoxidase-mediated protein modification via glycolaldehyde [34], which also contribute to the 'AGE-load' during diabetes. However, MG-H1 derived from MG, GO and 3-DG appear to be the major AGEs quantitatively measured in whole retina [23]. It has also been shown that MG-derived adducts accumulate around the retinal blood vessels of diabetic mice [28]. The current study indicates that Müller glia may be an important retinal cell-type for AGE formation in the diabetic retina. In the current study, galectin 3, a protein that binds AGEs and has been shown to be associated with blood retinal barrier dysfunction in diabetes [28], is upregulated in WT diabetic rats. Interestingly, GLO1 overexpression attenuates this diabetesinduced response. Such data indicate that further studies on the role of GLO1 in retinal vasopermeability during diabetes are warranted.

A range of AGE adducts may also bind to the receptor for AGEs (RAGE). RAGE is known to be highly expressed by Müller glia in the diabetic retina [30] and, although ligands such as S100B are important in this context, it is possible that receptor activation by MGderived AGEs could be playing an important role in diabetic retinopathy. Ongoing research is seeking to examine the pathophysiological implications of RAGE and MG-derived AGEs in Müller glia and their contribution to retinal lesions during diabetes.

GLO1 is part of an enzyme complex that requires reduced glutathione (GSH) as a co-factor and it is possible that following genetic overexpression its detoxification ability would be limited by available GSH. Previous studies have suggested that GSH and oxidised GSH do not change in diabetic retina, although enzymatic activity of superoxide dismutase, glutathione peroxidase, glutathione reductase and glutathione transferase are all reduced in diabetic rats compared with controls [35]. We have previously published that GLOI activity remains high in many tissues of these transgenic rats (including the eye) [19], and the observation that MG-related retinal AGEs are concomitantly reduced suggests that GSH is not limiting in the retina of these transgenic rats.

In addition to the glyoxalase-mediated detoxification, the aldose reductase (AR) pathway also plays a role in regulating intracellular levels of MG [36]. Indeed, MG acts as an aldehyde substrate of AR, which also requires GSH for enzymatic activity [37], although both enzyme systems appear to react differently to the diabetic milieu. For example in diabetic lens, GLO1 activity goes down, whereas AR is enhanced, and the AGE inhibitor pyridoxamine corrects both these responses [38]. In many tissues where GSH is high and AR activity low, the glyoxalase system may be the main detoxifier of MG [37]. Müller glia contain appreciable levels of intracellular GSH where it has antioxidant activity and is also linked to glutamate transporter activity [39]. Therefore, it is possible that AR and glyoxalase enzymes could both contribute to $\mathrm{MG}$ detoxification in the retina. How AR and glyoxalase enzymatic interactions contribute overall to retinal AGEs during diabetes requires further study.

Dysfunction of retinal Müller glia during diabetes has been described previously $[31,40]$ and manifests as upregulation of GFAP, cytokine expression and impaired protection against retinal excitotoxicity [41-43]. Winkler et al. have demonstrated that Müller glia exposed to high glucose conditions in vitro produce excess lactate, indicative of increased glycolytic flux [44], which would suggest enhanced susceptibility to MG and GO toxicity. In the current study, GLO1 overexpression protected against changes in GFAP production as a robust stress response by Müller glia associated with AGEs and associated lipid modifications [6]. In addition, GLOI overexpression also prevented the diabetes-linked mis-localisation of Kir4.1, which is a phenomenon also impacting on aquaporin 4 channel function in Müller glia [31, 45] and has already been linked to AGE accumulation [6]. Such upset of retinal $\mathrm{K}^{+}$clearance and hydration balance could promote oedema and neuronal hyper-excitation as diabetes progresses [31]. Kir4.1 has numerous arginine residues, although it remains unknown if this channel could be directly modified by MG with functional consequences for channel function or its normal association with $\alpha$-syntrophin [46].

One of the most significant findings of the current study is that $G L O 1$ overexpression protects against retinal capillary degeneration over 6 months of diabetes duration. We cannot be definitive if this phenomenon links completely to the observed Müller glia dysfunction established after 3 months of diabetes and ongoing after 6 months. However, such a link would seem likely as these glia play a 
major role in vascular integrity in the retina and disruption of the normal vascular-glial interactions, for example in ion exchange at the Müller glia end-feet [45], would impact on capillary degeneration. In addition, the importance of MG in endothelial cell damage during diabetes is established [22] and it has been previously demonstrated that GLO1 can regulate dysfunction of these cells during high glucose exposure in vitro $[17,18]$. In the current in vivo study, it is likely that GLO1 upregulation maintains the retinal capillary network by a combination of reducing MG-derived AGEs in Müller glia and endothelial cells.

In conclusion, our study demonstrates that elevating GLO1 offers protection against diabetic retinopathy and the data are consistent with a strong link between elevated MG, AGE formation and cell damage. Recently, it has also been demonstrated that the angiotensin type II receptor inhibitor candesartin can increase GLO1 activity, prevent formation of MG-derived AGEs and protect against key retinopathic lesions in vivo [47]. Therefore, there is considerable hope that enzymatic detoxification of AGE precursors could be a useful therapeutic target to prevent diabetic complications such as retinopathy.

Acknowledgements This work was funded by the Juvenile Diabetes Research Foundation (JDRF), Fight for Sight UK, a Dutch Diabetes Foundation Grant 2005.11.013 and Grant 2005.00.042 from the Diabetes Fonds Nederland. The authors would like to acknowledge the expert technical support of I. Vogels.

Contribution statement All authors contributed to analysis and interpretation of data, were involved in drafting the article and approved the final submission. In addition, AWS and CS contributed to the conception and design of the research and directed the overall study.

Duality of interest The authors declare that there is no duality of interest associated with this manuscript.

\section{References}

1. Stitt AW (2010) AGEs and diabetic retinopathy. Invest Ophthalmol Vis Sci 51:4867-4874

2. Beisswenger PJ, Szwergold BS, Yeo KT (2001) Glycated proteins in diabetes. Clin Lab Med 21:53-78, vi

3. Fosmark DS, Torjesen PA, Kilhovd BK et al (2006) Increased serum levels of the specific advanced glycation end product methylglyoxal-derived hydroimidazolone are associated with retinopathy in patients with type 2 diabetes mellitus. Metabolism 55:232-236

4. Yamaguchi M, Nakamura N, Nakano K et al (1998) Immunochemical quantification of crossline as a fluorescent advanced glycation endproduct in erythrocyte membrane proteins from diabetic patients with or without retinopathy. Diabet Med 15:458-462

5. Genuth S, Sun W, Cleary P et al (2005) Glycation and carboxymethyllysine levels in skin collagen predict the risk of future 10-year progression of diabetic retinopathy and nephropathy in the diabetes control and complications trial and epidemi- ology of diabetes interventions and complications participants with type 1 diabetes. Diabetes 54:3103-3111

6. Curtis TM, Hamilton R, Yong PH et al (2011) Muller glial dysfunction during diabetic retinopathy in rats is linked to accumulation of advanced glycation end-products and advanced lipoxidation end-products. Diabetologia 54:690-698

7. Stitt AW, Li YM, Gardiner TA, Bucala R, Archer DB, Vlassara H (1997) Advanced glycation end products (AGEs) co-localize with AGE receptors in the retinal vasculature of diabetic and of AGEinfused rats. Am J Pathol 150:523-531

8. Gardiner TA, Anderson HR, Stitt AW (2003) Inhibition of advanced glycation end-products protects against retinal capillary basement membrane expansion during long-term diabetes. J Pathol 201:328-333

9. Bhatwadekar A, Glenn JV, Figarola JL et al (2008) A new advanced glycation inhibitor, LR-90, prevents experimental diabetic retinopathy in rats. Br J Ophthalmol 92:545-547

10. Hammes HP, Alt A, Niwa T et al (1999) Differential accumulation of advanced glycation end products in the course of diabetic retinopathy. Diabetologia 42:728-736

11. Hammes HP, Du X, Edelstein D et al (2003) Benfotiamine blocks three major pathways of hyperglycemic damage and prevents experimental diabetic retinopathy. Nat Med 9:294-299

12. Rabbani N, Thornalley PJ (2010) Methylglyoxal, glyoxalase 1 and the dicarbonyl proteome. Amino Acids. doi:10.1007/s00726-010$0783-0$

13. Kilhovd BK, Giardino I, Torjesen PA et al (2003) Increased serum levels of the specific AGE-compound methylglyoxal-derived hydroimidazolone in patients with type 2 diabetes. Metabolism 52:163-167

14. Thornalley PJ, Langborg A, Minhas HS (1999) Formation of glyoxal, methylglyoxal and 3-deoxyglucosone in the glycation of proteins by glucose. Biochem J 344(Pt 1):109-116

15. Glomb MA, Lang G (2001) Isolation and characterization of glyoxalarginine modifications. J Agric Food Chem 49:1493-1501

16. Kuhla B, Luth HJ, Haferburg D, Boeck K, Arendt T, Munch G (2005) Methylglyoxal, glyoxal, and their detoxification in Alzheimer's disease. Ann N Y Acad Sci 1043:211-216

17. Shinohara M, Thornalley PJ, Giardino I et al (1998) Overexpression of glyoxalase-I in bovine endothelial cells inhibits intracellular advanced glycation endproduct formation and prevents hyperglycemia-induced increases in macromolecular endocytosis. J Clin Invest 101:1142-1147

18. Yao D, Taguchi T, Matsumura T et al (2007) High glucose increases angiopoietin-2 transcription in microvascular endothelial cells through methylglyoxal modification of mSin3A. J Biol Chem 282:31038-31045

19. Brouwers O, Niessen PM, Ferreira I et al (2011) Overexpression of glyoxalase-I reduces hyperglycemia-induced levels of advanced glycation end products and oxidative stress in diabetic rats. J Biol Chem 286:1374-1380

20. Brouwers O, Niessen PM, Haenen G et al (2010) Hyperglycaemiainduced impairment of endothelium-dependent vasorelaxation in rat mesenteric arteries is mediated by intracellular methylglyoxal levels in a pathway dependent on oxidative stress. Diabetologia 53:989-1000

21. Bento CF, Fernandes R, Ramalho J et al (2010) The chaperonedependent ubiquitin ligase CHIP targets HIF-1alpha for degradation in the presence of methylglyoxal. PLoS One 5:e15062

22. Queisser MA, Yao D, Geisler S et al (2010) Hyperglycemia impairs proteasome function by methylglyoxal. Diabetes 59:670-678

23. Karachalias N, Babaei-Jadidi R, Ahmed N, Thornalley PJ (2003) Accumulation of fructosyl-lysine and advanced glycation end products in the kidney, retina and peripheral nerve of streptozotocin-induced diabetic rats. Biochem Soc Trans $31: 1423-1425$ 
24. Denis U, Lecomte M, Paget C, Ruggiero D, Wiernsperger N, Lagarde M (2002) Advanced glycation end-products induce apoptosis of bovine retinal pericytes in culture: involvement of diacylglycerol/ceramide production and oxidative stress induction. Free Radic Biol Med 33:236-247

25. Miller AG, Smith DG, Bhat M, Nagaraj RH (2006) Glyoxalase I is critical for human retinal capillary pericyte survival under hyperglycemic conditions. J Biol Chem 281:11864-11871

26. Klaassen I, Hughes JM, Vogels IM, Schalkwijk CG, van Noorden CJ, Schlingemann RO (2009) Altered expression of genes related to blood-retina barrier disruption in streptozotocin-induced diabetes. Exp Eye Res 89:4-15

27. McVicar CM, Colhoun LM, Abrahams JL et al (2010) Differential modulation of angiogenesis by erythropoiesis-stimulating agents in a mouse model of ischaemic retinopathy. PLoS One 5:e11870

28. Canning P, Glenn JV, Hsu DK, Liu FT, Gardiner TA, Stitt AW (2007) Inhibition of advanced glycation and absence of galectin-3 prevent blood-retinal barrier dysfunction during short-term diabetes. Exp Diabetes Res 51837:1-10

29. Stitt A, Gardiner TA, Alderson NL et al (2002) The AGE inhibitor pyridoxamine inhibits development of retinopathy in experimental diabetes. Diabetes 51:2826-2832

30. Zong H, Ward M, Madden A et al (2010) Hyperglycaemiainduced pro-inflammatory responses by retinal Muller glia are regulated by the receptor for advanced glycation end-products (RAGE). Diabetologia 53:2656-2666

31. Pannicke T, Iandiev I, Wurm A et al (2006) Diabetes alters osmotic swelling characteristics and membrane conductance of glial cells in rat retina. Diabetes 55:633-639

32. Morcos M, Du X, Pfisterer F et al (2008) Glyoxalase-1 prevents mitochondrial protein modification and enhances lifespan in Caenorhabditis elegans. Aging Cell 7:260-269

33. Hughes JM, Kuiper EJ, Klaassen I et al (2007) Advanced glycation end products cause increased CCN family and extracellular matrix gene expression in the diabetic rodent retina. Diabetologia 50:1089-1098

34. Nagai R, Hayashi CM, Xia L, Takeya M, Horiuchi S (2002) Identification in human atherosclerotic lesions of GA-pyridine, a novel structure derived from glycolaldehyde-modified proteins. J Biol Chem 277:48905-48912

35. Obrosova IG, Fathallah L, Greene DA (2000) Early changes in lipid peroxidation and antioxidative defense in diabetic rat retina: effect of DL-alpha-lipoic acid. Eur J Pharmacol 398:139-146

36. Aguilera J, Prieto JA (2001) The Saccharomyces cerevisiae aldose reductase is implied in the metabolism of methylglyoxal in response to stress conditions. Curr Genet 39:273-283

37. Vander Jagt DL, Hassebrook RK, Hunsaker LA, Brown WM, Royer RE (2001) Metabolism of the 2-oxoaldehyde methylglyoxal by aldose reductase and by glyoxalase-I: roles for glutathione in both enzymes and implications for diabetic complications. Chem Biol Interact 130-132:549-562

38. Padival S, Nagaraj RH (2006) Pyridoxamine inhibits maillard reactions in diabetic rat lenses. Ophthalmic Res 38:294-302

39. Payet O, Maurin L, Bonne C, Muller A (2004) Hypoxia stimulates glutamate uptake in whole rat retinal cells in vitro. Neurosci Lett 356:148-150

40. Hammes HP, Federoff HJ, Brownlee M (1995) Nerve growth factor prevents both neuroretinal programmed cell death and capillary pathology in experimental diabetes. Mol Med 1:527-534

41. Mizutani M, Gerhardinger C, Lorenzi M (1998) Muller cell changes in human diabetic retinopathy. Diabetes 47:445-449

42. Fischer AJ, Scott MA, Ritchey ER, Sherwood P (2009) Mitogenactivated protein kinase-signaling regulates the ability of Muller glia to proliferate and protect retinal neurons against excitotoxicity. Glia 57:1538-1552

43. Shelton MD, Kern TS, Mieyal JJ (2007) Glutaredoxin regulates nuclear factor kappa-B and intercellular adhesion molecule in Muller cells: model of diabetic retinopathy. J Biol Chem 282:12467-12474

44. Winkler BS, Starnes CA, Sauer MW, Firouzgan Z, Chen SC (2004) Cultured retinal neuronal cells and Muller cells both show net production of lactate. Neurochem Int 45:311-320

45. Reichenbach A, Wurm A, Pannicke T, Iandiev I, Wiedemann P, Bringmann A (2007) Muller cells as players in retinal degeneration and edema. Graefes Arch Clin Exp Ophthalmol 245:627636

46. Connors NC, Adams ME, Froehner SC, Kofuji P (2004) The potassium channel Kir4.1 associates with the dystrophinglycoprotein complex via alpha-syntrophin in glia. J Biol Chem 279:28387-28392

47. Miller AG, Tan G, Binger KJ et al (2010) Candesartan attenuates diabetic retinal vascular pathology by restoring glyoxalase-I function. Diabetes 59:3208-3215 